\title{
Effects of the combination of vitamin $K$ and teriparatide on the bone metabolism in ovariectomized rats
}

\author{
NANA NAGURA ${ }^{1}$, JUN KOMATSU $^{1}$, HIDEAKI IWASE ${ }^{2}$, HIROSHI HOSODA $^{3}$, \\ OSAMU OHBAYASHI ${ }^{1}$, ISAO NAGAOKA ${ }^{3}$ and KAZUO KANEKO ${ }^{1}$ \\ ${ }^{1}$ Department of Medicine for Motor Organs, Juntendo University Graduate School of Medicine, Tokyo 113-8421; \\ ${ }^{2}$ Department of Bio-Engineering, Juntendo University Institute of Casualty Center, Izunokuni, \\ Shizuoka 410-2295; ${ }^{3}$ Department of Host Defense and Biochemical Research, \\ Juntendo University Graduate School of Medicine, Tokyo 113-8421, Japan
}

Received December 9, 2014; Accepted January 20, 2015

DOI: $10.3892 /$ br.2015.431

\begin{abstract}
The purpose of the present study was to evaluate the combined effects of vitamin $\mathrm{K}(\mathrm{VK})$ and teriparatide (TPTD) on bone mineral density (BMD), mechanical strength and other parameters for bone metabolism using a rat ovariectomized osteoporosis model. Ovariectomized female Sprague-Dawley rats were administered with VK (an oral dose of $30 \mathrm{mg} / \mathrm{kg} / \mathrm{day}$ ), TPTD (a subcutaneous dose of $30 \mu \mathrm{g} / \mathrm{kg}$, three times a week) or a combination for 8 weeks. Thereafter, serum levels of $\gamma$-carboxylated osteocalcin (Gla-OC) were quantitated by ELISA; BMD and mechanical strength were measured by computed tomography and biomechanical testing, respectively at the femoral metaphysis. Additionally, histomorphometry was performed using the toluidine blue-stained coronal sections of distal femur. The combination of VK and TPTD clearly increased the serum levels of Gla-OC (a specific marker for bone formation) and osteoblast surface (the number of osteoblasts attaching with the surface of cancellous bone), compared to VK or TPTD alone. In addition, the combination of the two agents improved the BMD and bone strength of the femur in the ovariectomized rats, compared to VK or TPTD alone. Taken together, these findings suggest that the treatment with VK and TPTD may have a therapeutic advantage over VK or TPTD monotherapy for postmenopausal osteoporosis, possibly by enhancing the bone formation through the actions on OC and osteoblasts.
\end{abstract}

Correspondence to: Professor Isao Nagaoka, Department of Host Defense and Biochemical Research, Juntendo University Graduate School of Medicine, 2-1-1 Hongo, Tokyo 113-8421, Japan E-mail: nagaokai@juntendo.ac.jp

Key words: vitamin K, teriparatide, bone metabolism, osteocalcin, ovariectomized rats

\section{Introduction}

Postmenopausal osteoporosis is a degenerative disease characterized by reduced bone mass, as estrogen deficiency during menopause enhances the bone turnover, in which bone degradation exceeds bone formation. Postmenopausal osteoporosis leads to bone fragility and a consequent increase in fracture risk. To reduce the risk of fracture, it is important to improve bone strength clinically using several agents, including bisphosphonate, vitamin $\mathrm{D}$, vitamin $\mathrm{K}(\mathrm{VK})$ and teriparatide (TPTD). In addition, the combination therapy with these agents can increase the effectiveness of treatment in certain cases and is now the subject of extensive investigation $(1,2)$.

$\mathrm{VK}$ is essential for the $\gamma$-carboxylation of osteocalcin (OC), a major non-collagenous bone protein, synthesized by osteoblasts. $\gamma$-carboxylated OC (Gla-OC) is the most active form and stimulates osteoblasts to enhance mineralization $(3,4)$. Previously, it has been reported that VK binds to the steroid and xenobiotic receptor and modulates the gene transcription in human osteoblastic cells $(5,6)$. Additionally, VK plays roles not only in enhancing bone formation, but also in suppressing bone degradation $(7,8)$. Clinically, VK has been shown to prevent bone loss and fractures in postmenopausal females with osteoporosis (9-12).

By contrast, an intermittent injection of low-dose parathyroid hormone-derived peptide (TPTD) can effectively stimulate bone formation by increasing osteoblast number and activity via the promotion of the differentiation and survival of osteoblasts, thereby increasing trabecular bone mass and strength (13-17). In fact, clinical trials show that TPTD increases bone mineral density (BMD) and decreases the fracture risk in females with postmenopausal osteoporosis (18-20).

The VK-dependent $\gamma$-carboxylation of OC was recently reported to enhance the efficacy of TPTD against bone repair in a rat osteotomy model (21). Thus, we hypothesized that the combination of VK and TPTD may improve the bone metabolism in osteoporosis by increasing the bone formation but suppressing the bone degeneration. Therefore, in the present study, the effects of the combination of VK and TPTD on BMD, mechanical strength and other parameters for bone metabolism were examined in a rat ovariectomized osteoporosis model. 


\section{Materials and methods}

Animals. All the procedures were carried out according to the Institutional Animal Care and Committee Guide of Juntendo University School of Medicine (Tokyo, Japan). Twenty five female Sprague Dawley rats (13 weeks old; Japan SLC, Tokyo, Japan) were used. The rats were housed in a controlled environment at $25 \pm 1^{\circ} \mathrm{C}$ with a 12 -h on/off light cycle and free access to deionized water.

Experiment protocols. After adaptation to the new environment for one week, rats were assigned to five groups: The sham, ovariectomy (OVX), VK, TPTD and VK + TPTD groups. At 14 weeks of age, bilateral OVX was performed from a dorsal approach under inhalation anesthesia of halothane for 20 rats belonging to the OVX, VK, TPTD and VK + TPTD groups. Five rats were subjected to the sham operation in which the ovaries were exteriorized but not removed. VK $(30 \mathrm{mg} / \mathrm{kg} /$ day $)$ was orally administered to rats in the VK and VK + TPTD groups by feeding with a VK-deficient diet (CLEA Japan, Inc., Tokyo, Japan) containing $65 \mathrm{mg}$ VK (menaquinone MK-4; Eisai Co., Ltd., Tokyo, Japan) and $0.5 \mathrm{~g}$ calcium per $100 \mathrm{~g}$ diet. Rats in the other groups were fed the VK-deficient diet containing $0.5 \mathrm{~g}$ calcium per $100 \mathrm{~g}$ diet. Teriparatide acetate (corresponding to the $\mathrm{N}$-terminal 1-34 amino acid residues of human parathyroid hormone consisting of 84 amino acids; Asahi Kasei Pharma Corporation, Tokyo, Japan) was dissolved in saline containing $0.1 \%$ rat serum albumin and injected subcutaneously three times a week at a dose of $30 \mu \mathrm{g} / \mathrm{kg}$ in the TPTD and VK + TPTD groups, and rats in the other groups were injected with saline as a vehicle. After the administration of VK and TPTD for 8 weeks, rats were anesthetized by intraperitoneal injection of pentobarbital sodium $(50 \mathrm{mg} / \mathrm{kg})$ and blood samples were obtained from the femoral vein. Following sacrifice by exsanguination, bilateral femurs, from which muscles were detached, were collected from every rat. Serum was separated by centrifugation of blood at $770 \mathrm{x} \mathrm{g}$ for $20 \mathrm{~min}$ at $4^{\circ} \mathrm{C}$ and stored at $-80^{\circ} \mathrm{C}$. The right femurs were stored at $-80^{\circ} \mathrm{C}$ for BMD measurement and biomechanical testing; the left femurs were immersed in $70 \%$ ethanol solution for histomorphometry.

Biomarker assays. Serum levels of Gla-OC and Glu-OC (the non $\gamma$-carboxylated form of OC) were measured using an enzyme-linked immunosorbent assay (Rat Gla-Osteocalcin High Sensitive EIA and Rat Glu-Osteocalcin High Sensitive EIA kits; Takara Bio Inc., Shiga, Japan). Serum levels of C-terminal telopeptide of type I collagen (CTX-I) was measured by an enzyme-linked immunosorbent assay (RatLaps EIA; Immunodiagnostic Systems Ltd., Boldon, UK).

BMD assay. The BMD of femurs was measured by X-ray computed tomography (Latheta LCT-200; Hitachi Aloka Medical, Ltd., Tokyo, Japan). BMD of diaphysis was measured at the mid-diaphysis position of femurs and BMD of metaphysis was measured at $1.5 \mathrm{~mm}$ proximal to the growth plate in the condyle of femurs.

Biomechanical testing. Biomechanical properties were measured by a three-point bending test at mid-diaphysis and a compression test at metaphysis using a servo-hydraulic fatigue-testing instrument (Servopulser; Shimadzu Co., Kyoto, Japan). The mid-diaphysis was placed on the two supports that were $20 \mathrm{~mm}$ apart. The load of a three-point bending test was applied in the anteroposterior direction midway between the two supports. Load displacement curves were recorded at a crosshead speed of $6 \mathrm{~mm} / \mathrm{sec}$. Alternatively, metaphysis was placed on the test apparatus with the lateral side up. Compression load was applied on the specimens from the lateral aspect to the medial aspect. Load displacement curves were recorded at a crosshead speed of $6 \mathrm{~mm} / \mathrm{sec}$. The maximum load, breaking energy and stiffness were calculated using a load-displacement curve.

Histomorphometry. The left femurs were fixed and embedded in glycol methacrylate. Coronal sections of distal femur were cut at a thickness of $20 \mu \mathrm{m}$ and stained with toluidine blue for histomorphometric analyses. Measurements were performed in the secondary cancellous bone region from 0.2 to $2.25 \mathrm{~mm}$ proximal to the growth plate using a semi-automatic image analysis system (OsteoMetrics, Inc., Decatur, GA, USA) and the primary parameters, such as tissue volume (TV), bone volume (BV), bone surface (BS), osteoblast surface (Ob.S) and osteoclast surface (Oc.S), were determined. These data were used to calculate the following parameters: BV/TV, Ob.S/BS and Oc.S/BS.

Statistical analysis. All the data are expressed as mean \pm standard deviation. Multiple comparisons of data among the groups were performed by analysis of variance with Tukey's post hoc test. $\mathrm{P}<0.05$ was considered to indicate a statistically significant difference.

\section{Results}

Biomarker assays. Fig. 1 shows the serum levels of Gla-OC, Glu-OC and CTX-I. The levels of Gla-OC and CTX-I in the OVX group were significantly increased compared to the Sham group $(\mathrm{P}<0.05)$, although there was no significant change in the serum level of Glu-OC. Compared to the OVX group, the Gla-OC level was slightly increased in the VK group and significantly increased in the TPTD group $(\mathrm{P}<0.01)$ (Fig. 1A). Additionally, the Gla-OC level was further increased in the $\mathrm{VK}+\mathrm{TPTD}$ group compared to the VK and TPTD groups $(\mathrm{P}<0.05)$.

Notably, the Glu-OC level was significantly decreased in the VK group $(\mathrm{P}<0.01)$, but increased in the TPTD group $(\mathrm{P}<0.05)$ compared to the OVX group (Fig. 1B). The Glu-OC level decreased in the VK + TPTD group compared to the TPTD group, although there was no significant difference between the two groups.

The CTX-I level was decreased in the VK group compared to the OVX group (Fig. 1C). Additionally, the CTX-I level was significantly increased in the TPTD group compared to the Sham group. Of note, the CTX-I level was increased in the TPTD group compared to the VK group but decreased in the $\mathrm{VK}+\mathrm{TPTD}$ group compared to the TPTD group.

Assay of BMD. Fig. 2 shows the BMD of the femoral metaphysis and diaphysis. The BMD of the femoral metaphysis in the OVX 
A

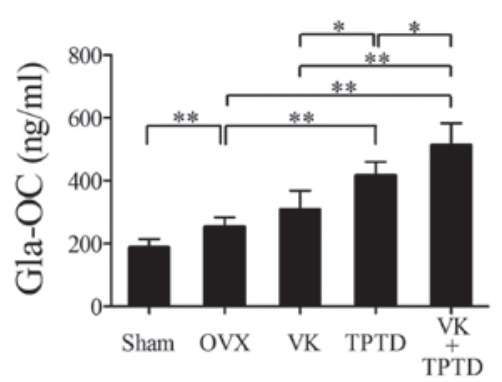

B

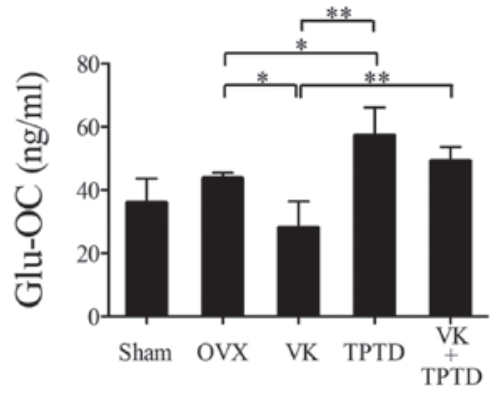

C

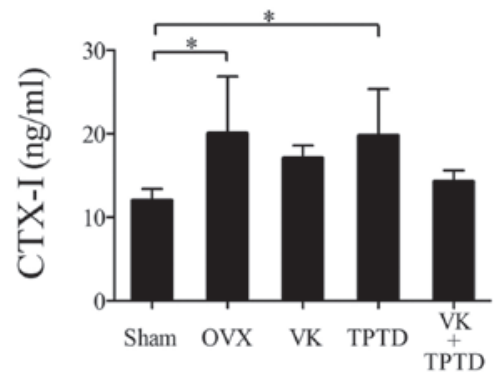

Figure. 1. Serum levels of $\gamma$-carboxylated osteocalcin (Gla-OC), Glu-OC and C-terminal telopeptide of type I collagen (CTX-I) in rats administered with vitamin $\mathrm{K}$ (VK) and teriparatide (TPTD). Rats were assigned to the Sham, ovariectomy (OVX), VK, TPTD and VK + TPTD groups (five rats in each group). Bilateral OVX was performed in rats belonging to the OVX, VK, TPTD and VK + TPTD groups. VK (30 mg/kg/day) was orally administered to rats in the VK and VK + TPTDP groups. TPTD was injected subcutaneously three times a week at a dose of $30 \mu \mathrm{g} / \mathrm{kg}$ in the TPTD and VK + TPTD groups. After the administration of VK and TPTD for 8 weeks, sera were prepared for measurement of (A) Gla-OC, (B) Glu-OC and (C) CTX-I. Data are expressed as mean \pm standard deviation and compared among the groups. ${ }^{*} \mathrm{P}<0.05$ and ${ }^{* *} \mathrm{P}<0.01$.

group was significantly decreased compared to the Sham group $(\mathrm{P}<0.05)$ (Fig. 2A). Of note, the decreased level of BMD (OVX group) was not changed by VK administration but significantly increased by TPTD administration $(\mathrm{P}<0.01)$. Furthermore, BMD in the combination of VK and TPTD group was significantly increased compared to the $\mathrm{VK}$ group $(\mathrm{P}<0.01)$ and increased compared to the TPTD group. By contrast, BMD of the femoral diaphysis was not significantly different among the five groups (Sham, OVX, VK, TPTD and VK + TPTD) (Fig. 2B).

Biomechanical testing. Fig. 3 shows the results of biomechanical testing of metaphysis. The maximum load and breaking energy in the OVX group were lower compared to the Sham group (Fig. 3A and B). Notably, the maximum load and breaking energy in the TPTD group were increased compared to the OVX group and breaking energy in the VK group was increased compared to the OVX group (Fig. 3A and B). Additionally, in the VK + TPTD group, the maximum load and breaking energy were significantly increased compared to the OVX group $(\mathrm{P}<0.05)$.
A

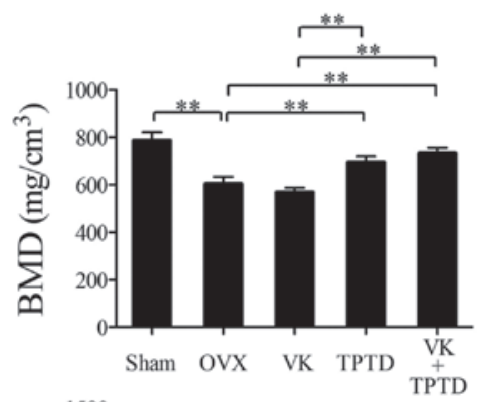

B

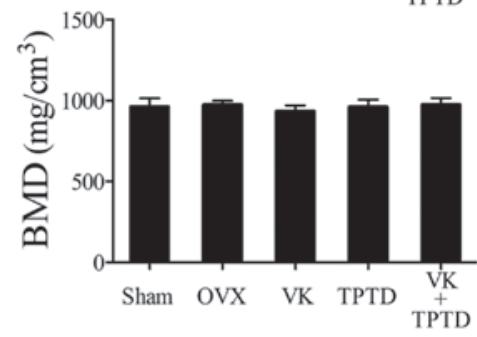

Figure 2. Bone mineral density (BMD) of the femoral metaphysis and diaphysis of rats administered with vitamin $\mathrm{K}$ (VK) and teriparatide (TPTD). After the administration of VK and TPTD for 8 weeks, BMD was measured using the (A) femoral metaphysis and (B) diaphysis of rats belonging to the Sham, ovariectomy (OVX), VK, TPTD and VK + TPTD groups (five rats in each group). Data are expressed as mean \pm standard deviation and compared among the groups. ${ }^{* *} \mathrm{P}<0.01$.

A

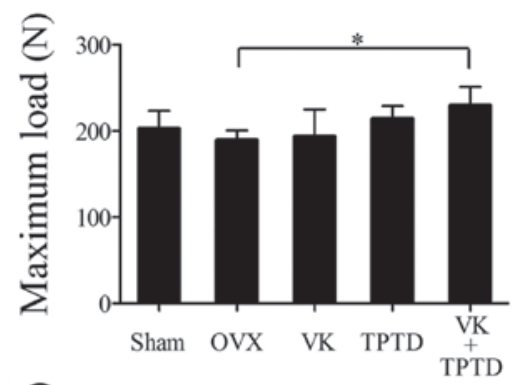

B

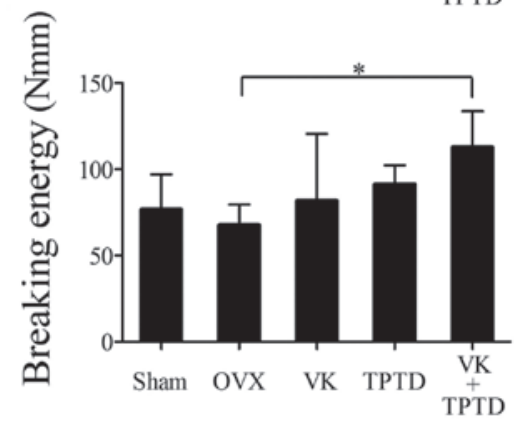

C

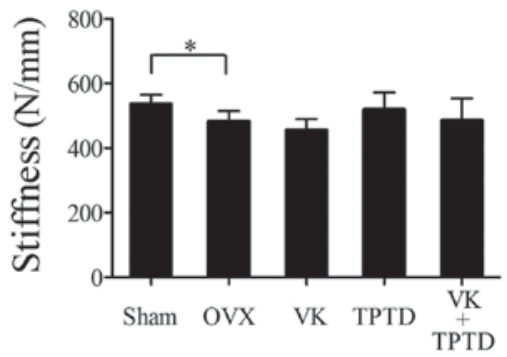

Figure 3. Biomechanical properties of the femoral metaphysis in rats administered with vitamin $\mathrm{K}$ (VK) and teriparatide (TPTD). After the administration of VK and TPTD for 8 weeks, the biomechanical properties of (A) maximum load, (B) breaking energy and (C) stiffness were measured by a compression test at the femoral metaphysis of rats belonging to the Sham, ovariectomy (OVX), VK, TPTD and VK + TPTD groups (five rats in each group). Data are expressed as mean \pm standard deviation and compared among the groups. ${ }^{*} \mathrm{P}<0.05$. 
A

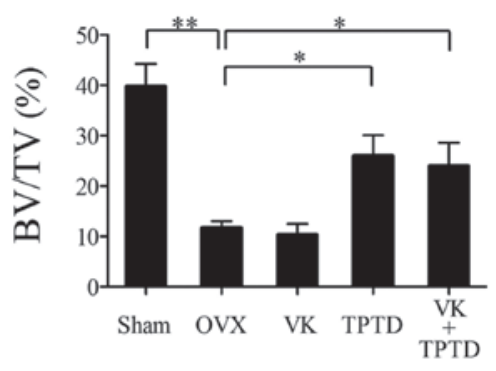

B

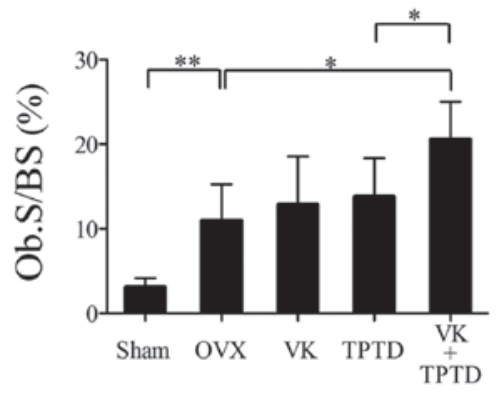

C

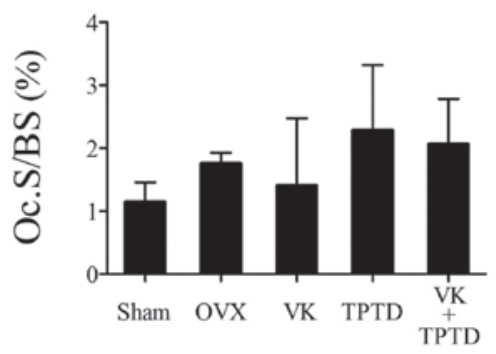

Figure 4. Histomorphometry of the femoral metaphysis in rats administered with vitamin $\mathrm{K}$ (VK) and teriparatide (TPTD). Histomorphometry of (A) bone volume (BV)/tissue volume (TV), (B) osteoblast surface (Ob.S)/bone surface (BS) and (C) osteoclast surface (Oc.S)/BS were measured at the femoral metaphysis of rats belonging to the Sham, ovariectomy (OVX), VK, TPTD and VK + TPTD groups (five rats in each group). Data are expressed as mean \pm standard deviation and compared among the groups. ${ }^{*} \mathrm{P}<0.05$ and ${ }^{* *} \mathrm{P}<0.01$.

The stiffness of the metaphysis was significantly decreased in the OVX group compared to the Sham group $(\mathrm{P}<0.05)$ (Fig. 3C). The decreased level of the stiffness was further decreased in the VK group but increased in the TPTD group compared to the OVX group (Fig. 3C). Notably, the increased level of stiffness in the TPTD group was decreased by the combination with VK (the VK + TPTD group).

Similar to the finding in BMD (Fig. 2B), the biomechanical parameters of femoral diaphysis (maximum load, breaking energy and stiffness) were not significantly different among the five groups (Sham, OVX, VK, TPTD and VK + TPTD) (data not shown).

Bone histomorphometry. Fig. 4 shows the results of bone histomorphometric analysis of femoral metaphysis. BV/TV was significantly decreased in the OVX compared to the Sham group $(\mathrm{P}<0.01)$ (Fig. 4A). The decreased level of BV/TV was significantly increased in the TPTD group $(\mathrm{P}<0.05)$, but not changed in the VK group. In addition, the decreased level of BV/TV was significantly increased in the VK + TPTD group compared to the OVX group $(\mathrm{P}<0.05)$, although there was no significant difference between the TPTD and VK + TPTD groups.
Ob.S/BS was significantly increased in the OVX group compared to the Sham group (Fig. 4B). Additionally, Ob.S/BS was increased in the VK and TPTD groups compared to the OVX group. Notably, Ob.S/BS was significantly increased in the VK + TPTD group compared to the OVX group and TPTD group $(\mathrm{P}<0.05)$.

Oc.S/BS was slightly increased in the OVX group compared to the Sham group (Fig. 4C). Of note, Oc.S/BS was decreased in the VK group compared to the OVX group but increased in the TPTD group compared to the OVX group. Notably, Oc.S/BS was decreased in the VK + TPTD group compared to the TPTD group.

\section{Discussion}

In the present study, the effects of a combination of VK and TPTD on BMD, mechanical strength and other parameters were examined for bone metabolism using a rat ovariectomized osteoporosis model. The combination of VK and TPTD markedly increased the serum levels of Gla-OC (a specific marker for bone formation) and Ob.S (the number of osteoblasts attaching with the surface of cancellous bone) compared to VK or TPTD. In addition, the combination of VK and TPTD improved the BMD and bone strength of the femur in the estrogen-deficient (ovariectomized) osteoporosis of rats. These findings suggest that the treatment with VK and TPTD may have a therapeutic advantage over VK or TPTD monotherapy for postmenopausal osteoporosis, possibly by enhancing the bone formation through the increase of $\mathrm{OC}$ and the activation of osteoblasts.

Ovariectomized animals have been widely used as an experimental model of osteoporosis. This model is highly reproducible and characterized by loss of bone mass due to the high bone turnover, in which the bone formation and degradation are enhanced due to estrogen deficiency (22). In the present study, OVX caused the loss of mineral and mechanical properties in the metaphysis of femurs, but did not affect these parameters in the diaphysis of femurs (Figs. 2 and 3). Consistent with this observation, OVX reportedly shows the loss of cancellous (metaphysis) bone compared to cortical (diaphysis) bone (23). In addition, the present study identified that OVX induced a significant increase in the serum levels of Gla-OC (a bone formation parameter) and CTX-I (a bone degradation parameter), confirming the enhanced bone turnover in the model following estrogen deficiency.

Administration of VK increased Gla-OC and decreased CTX-I in sera. Similarly, VK modulated Ob.S/BS and Oc.S/BS in the histomorphometric parameters. Although these changes were small, the present results suggest that VK increases the bone formation but suppresses the bone degradation in an osteoporosis model, as previously reported for the effect of VK $(7,8,24,25)$. Of note, VK administration significantly decreases Glu-OC but increases Gla-OC in sera, possibly by acting on the OC metabolism. Consistent with this, it is reported that non- or undercarboxylated osteocalcin (Glu-OC) is decreased, but Gla-OC is increased in sera of individuals administered with VK (9).

TPTD effectively promotes bone formation and enhances cancellous bone mass by stimulating the number and activity of trabecular osteoblasts in ovariectomized rats (26-28). The present study showed that the administration of TPTD 
promotes the bone formation as indicated by increasing Gla-OC in sera and Ob.S/BS in the histomorphometry. In addition, TPTD administration increased not only Gla-OC but also Glu-OC in sera. Thus, it is possible that TPTD enhances the synthesis of OC itself via the stimulation of osteoblasts, thereby increasing Glu-OC. The effects of TPTD on bone degradation are unclearly defined; however, the present study has indicated that TPTD increased Oc.S/BS in histomorphometry but did not change CTX-I level in sera.

The combination administration of VK and TPTD increased Gla-OC and Ob.S/BS compared to TPTD treatment alone. By contrast, the combination administration reduced the increased levels of CTX-I compared to TPTD treatment alone. These results suggest that the combination of VK and TPTD promotes the bone formation but suppresses bone degradation. Of note, VK reduced the CTX-I and Oc.S/BS compared to OVX and the combination of VK and TPTD decreased these parameters compared to TPTD alone (Figs. 1 and 4). These results suggest that VK suppresses the bone degradation when administered alone or in combination with TPTD.

In the present study, TPTD administration significantly increased the BMD and slightly enhanced the bone strength (maximum load and breaking energy). These results are consistent with previous studies showing that TPTD restores lost bone mass and enhances bone strength in ovariectomized rat models (29-32). By contrast, VK administration increased the breaking energy but did not increase the BMD. Consistently, previous studies indicated that VK improves bone strength without necessarily increasing BMD (33). These effects of VK are considered to be due to the increase of active (carboxylated) OC and lysyl oxidase-mediated enzymatic cross-linking of collagen, thereby improving bone strength (elasticity and toughness) without significantly affecting BMD (34-36). Notably, the combination of VK and TPTD increased the BMD compared to VK alone. In addition, the combination further increased bone strength (breaking energy and maximum load) compared to VK or TPTD alone. These results suggest that the combination of VK and TPTD is effective in increasing not only BMD but also bone strength. Notably, the administration of VK or the combination of VK and TPTD decreased the stiffness compared to OVX or TPTD alone, respectively. These observations may suggest that VK possibly makes the cancellous bone pliable. Thus, the combination of VK and TPTD is expected to improve not only the bone strength, but also the bone quality.

In conclusion, the present study revealed that the combination of VK and TPTD promoted the bone formation and improved the BMD and bone strength of the femurs in ovariectomized rats. Thus, the combination administered is expected to be effective in preventing fractures in postmenopausal osteoporosis. Further studies are required to clarify the molecular mechanisms for the cooperative action of VK and TPTD on the bone metabolism in the postmenopausal osteoporosis.

\section{Acknowledgements}

The authors would like to thank Mr. Masahiro Miyazaki (Department of Bio-Engineering, Juntendo University Institute of Casualty Center, Shizuoka, Japan) for the technical assistance in the research. The authors are also indebted to Eisai
Pharmaceutical, Japan and Asahi Kasei Pharma Co., Ltd., for their supplies of VK and TPTD.

\section{References}

1. Orimo H, Nakamura T, Fukunaga M, et al: Effects of alendronate plus alfacalcidol in osteoporosis patients with a high risk of fracture: The Japanese Osteoporosis Intervention Trial (JOINT)-02. Curr Med Res Opin 27: 1273-1284, 2011.

2. Ushiroyama T, Ikeda A, Sakai M, Higashiyama T and Ueki M: Effects of the combined use of calcitonin and 1 alpha-hydroxycholecalciferol on vertebral bone loss and bone turnover in women with postmenopausal osteopenia and osteoporosis: A prospective study of long-term and continuous administration with low dose calcitonin. Maturitas 40: 229-238, 2001

3. Kobayashi M, Hara K and Akiyama Y: Effects of vitamin K2 (menatetrenone) and alendronate on bone mineral density and bone strength in rats fed a low-magnesium diet. Bone 35 : 1136-1143, 2004

4. Price PA: Vitamin K-dependent formation of bone Gla protein (osteocalcin) and its function. Vitam Horm 42: 65-108, 1985.

5. Tabb MM, Sun A, Zhou C, et al: Vitamin K2 regulation of bone homeostasis is mediated by the steroid and xenobiotic receptor SXR. J Biol Chem 278: 43919-43927, 2003.

6. Igarashi M, Yogiashi Y, Mihara M, Takada I, Kitagawa H and Kato S: Vitamin K induces osteoblast differentiation through pregnane $X$ receptor-mediated transcriptional control of the Msx2 gene. Mol Cell Biol 27: 7947-7954, 2007.

7. Akiyama Y, Hara K, Kobayashi M, Tomiuga T and Nakamura T: Inhibitory effect of vitamin K2 (menatetrenone) on bone resorption in ovariectomized rats: A histomorphometric and dual energy X-ray absorptiometric study. Jpn J Pharmacol 80: 67-74, 1999.

8. Hara K, Akiyama Y, Nakamura T, Murota S and Morita I: The inhibitory effect of vitamin K2 (menatetrenone) on bone resorption may be related to its side chain. Bone 16: 179-184, 1995.

9. Shiraki M, Shiraki Y, Aoki C and Miura M: Vitamin K2 (menatetrenone) effectively prevents fractures and sustains lumbar bone mineral density in osteoporosis. J Bone Miner Res 15: 515-521, 2000.

10. Inoue $T$, Fujita $T$, Kishimoto $H$, Makino $T$, Nakamura $T$, Nakamura T, Sato T and Yamazaki K: Randomized controlled study on the prevention of osteoporotic fractures (OF study): A phase IV clinical study of $15-\mathrm{mg}$ menatetrenone capsules. J Bone Miner Metab 27: 66-75, 2009.

11. Iwamoto J: Vitamin $\mathrm{K}_{2}$ therapy for postmenopausal osteoporosis. Nutrients 6: 1971-1980, 2014.

12. Feskanich D, Weber P, Willett WC, Rockett H, Booth SL and Colditz GA: Vitamin $\mathrm{K}$ intake and hip fractures in women: A prospective study. Am J Clin Nutr 69: 74-79, 1999.

13. Tam CS, Heersche JN, Murray TM and Parsons JA: Parathyroid hormone stimulates the bone apposition rate independently of its resorptive action: Differential effects of intermittent and continuous administration. Endocrinology 110: 506-512, 1982.

14. Rubin MR, Cosman F, Lindsay R and Bilezikian JP: The anabolic effects of parathyroid hormone. Osteoporos Int 13: 267-277, 2002.

15. Rosen CJ: The role of parathyroid hormone in the management of osteoporosis. Horm Res 64 (Suppl 2): 81-85, 2005.

16. Kishi T, Hagino H, Kishimoto $\mathrm{H}$ and Nagashima $\mathrm{H}$ : Bone responses at various skeletal sites to human parathyroid hormone in ovariectomized rats: Effects of long-term administration, withdrawal, and readministration. Bone 22: 515-522, 1998.

17. Okimoto N, Tsurukami H, Okazaki Y, Nishida S, Sakai A, Ohnishi H, Hori M, Yasukawa K and Nakamura T: Effects of a weekly injection of human parathyroid hormone (1-34) and withdrawal on bone mass, strength, and turnover in mature ovariectomized rats. Bone 22: 523-531, 1998.

18. Neer RM, Arnaud CD, Zanchetta JR, et al: Effect of parathyroid hormone (1-34) on fractures and bone mineral density in postmenopausal women with osteoporosis. N Engl J Med 344: 1434-1441, 2001.

19. Miyauchi A, Matsumoto T, Sugimoto T, Tsujimoto M, Warner MR and Nakamura T: Effects of teriparatide on bone mineral density and bone turnover markers in Japanese subjects with osteoporosis at high risk of fracture in a 24-month clinical study: 12-month, randomized, placebo-controlled, double-blind and 12-month open-label phases. Bone 47: 493-502, 2010. 
20. Dempster DW, Cosman F, Kurland ES, et al: Effects of daily treatment with parathyroid hormone on bone microarchitecture and turnover in patients with osteoporosis: A paired biopsy study. J Bone Miner Res 16: 1846-1853, 2001.

21. Shimizu T, Takahata M,Kameda Y,Hamano H,Ito T,Kimura-Suda H, Todoh M, Tadano S and Iwasaki N: Vitamin K-dependent carboxylation of osteocalcin affects the efficacy of teriparatide (PTH(1-34)) for skeletal repair. Bone 64: 95-101, 2014.

22. Turner RT, Vandersteenhoven JJ and Bell NH: The effects of ovariectomy and 17 beta-estradiol on cortical bone histomorphometry in growing rats. J Bone Miner Res 2: 115-122, 1987.

23. Kalu DN: The ovariectomized rat model of postmenopausal bone loss. Bone Miner 15: 175-191, 1991.

24. Koshihara Y and Hoshi K: Vitamin K2 enhances osteocalcin accumulation in the extracellular matrix of human osteoblasts in vitro. J Bone Miner Res 12: 431-438, 1997.

25. Iwasaki Y, Yamato H, Murayama H, Takahashi T, Ezawa I, Kurokawa K and Fukagawa M: Menatetrenone prevents osteoblast dysfunction in unilateral sciatic neurectomized rats. Jpn J Pharmacol 90: 88-93, 2002.

26. Hori M, Uzawa T, Morita K, Noda T, Takahashi H and Inoue J: Effect of human parathyroid hormone (PTH(1-34)) on experimental osteopenia of rats induced by ovariectomy. Bone Miner 3 : 193-199, 1988

27. Fox J, Miller MA, Newman MK, Metcalfe AF, Turner CH, Recker RR and Smith SY: Daily treatment of aged ovariectomized rats with human parathyroid hormone (1-84) for 12 months reverses bone loss and enhances trabecular and cortical bone strength. Calcif Tissue Int 79: 262-272, 2006

28. Iwaniec UT, Mosekilde L, Mitova-Caneva NG, Thomsen JS and Wronski TJ: Sequential treatment with basic fibroblast growth factor and PTH is more efficacious than treatment with PTH alone for increasing vertebral bone mass and strength in osteopenic ovariectomized rats. Endocrinology 143: 2515-2526, 2002.
29. Hock JM, Gera I, Fonseca J and Raisz LG: Human parathyroid hormone-(1-34) increases bone mass in ovariectomized and orchidectomized rats. Endocrinology 122: 2899-2904, 1988.

30. Alexander JM, Bab I, Fish S, et al: Human parathyroid hormone 1-34 reverses bone loss in ovariectomized mice. J Bone Miner Res 16: 1665-1673, 2001.

31. Li M, Liang H, Shen Y and Wronski TJ: Parathyroid hormone stimulates cancellous bone formation at skeletal sites regardless of marrow composition in ovariectomized rats. Bone 24: 95-100, 1999.

32. Wronski TJ, Yen CF, Qi H and Dann LM: Parathyroid hormone is more effective than estrogen or bisphosphonates for restoration of lost bone mass in ovariectomized rats. Endocrinology 132: 823-831, 1993.

33. Matsumoto T, Miyakawa T and Yamamoto D: Effects of vitamin $\mathrm{K}$ on the morphometric and material properties of bone in the tibiae of growing rats. Metabolism 61: 407-414, 2012.

34. Saito M and Marumo K: Collagen cross-links as a determinant of bone quality: A possible explanation for bone fragility in aging, osteoporosis, and diabetes mellitus. Osteoporos Int 21: 195-214, 2010.

35. Iwamoto J, Sato Y, Takeda T and Matsumoto H: Bone quality and vitamin $\mathrm{K} 2$ in type 2 diabetes: Review of preclinical and clinical studies. Nutr Rev 69: 162-167, 2011.

36. Shiraishi A, Higashi S, Masaki T, Saito M, Ito M, Ikeda S and Nakamura T: A comparison of alfacalcidol and menatetrenone for the treatment of bone loss in an ovariectomized rat model of osteoporosis. Calcif Tissue Int 71: 69-79, 2002. 